\title{
Research on Chinese Industrial Upgrading under Trade Integration
}

\author{
Hong Lv \\ Nanyang Institute of Technology, Nanyang, Henan, 473004
}

Keywords: Industrial Upgrading, Trade Integration, Chinese Industry

\begin{abstract}
Based on the theoretical framework of industrial upgrading in global production networks, this paper studies the mechanisms and influencing factors of the related industries in their continuous promotion in the process of global division of labor, assesses the status quo of the upgrading of relevant industry networks in China, and on this basis, Measures to Promote the Upgrading of Related Industrial Networks in China.
\end{abstract}

\section{Introduction}

At present, with the deepening of international trade and the international division of labor, the world economy is rapidly merging into a highly integrated economy. In this process, the global production network led by multinational corporations has become an increasingly important new production organization way. The global production network theory model has become an important basis for MNCs to formulate their global strategies and has an important impact on the active participation of enterprises and related industries in developing countries in international economic cooperation and regional economic development. Meanwhile, the global production network as a new theoretical analysis Tools can be used to analyze the current situation of international division of labor and have important theoretical guiding significance for the enterprises in developing countries to effectively integrate into the global economy and continuously upgrade their industries. From the perspective of global production networks, we can see that the global competitive environment has undergone major changes. The competition among countries shows not only the competition among industries, but also the competition among different production processes in the same industry, namely, who occupy the core or key link in the industrial value chain, who has won the most favorable competitive advantage. Therefore, China needs to re-examine and adjust the corresponding industrial development policies and business strategies to promote industrial upgrading, enhance its position in the global value chain system, further enhance its international competitiveness and obtain more economic benefits.

\section{Global Production Network Motivation and Determinants Analysis}

For a long time, in order to cope with the pressure of global competition, multinational corporations are committed to finding ways to reduce their production costs. The abundant labor resources of developing countries and the low wage level continuously push them to pass the processing links of manufacturing and labor-intensive products The way to set up overseas production bases should be put in developing countries or regions so that MNCs themselves can enhance their competitiveness in the market. In this process, attracting multinational corporations is the different competitive advantages of different countries and regions. For example, most developing countries have the comparative advantage of labor force and are allocated to labor-intensive production. Developed countries have outstanding advantages in capital, technology and marketing. They are allocated to such aspects as design, brand and marketing. The carrier or medium that organizes enterprises from different countries and regions together is the global production network. In a production network, the dominant strategic position and play an organizational role of the network initiated by the business called the leading manufacturers or brand manufacturers, they are often multinational 
corporations. In a specific production network, from the perspective of the governance model of economic organizations, the global production network is different from the traditional binary governance model in that it is not a purely internal MNC governance model, and certainly not a market governance mode. In other words, the leader in the production network and other manufacturers not through the most flexible employment relationship, nor through loose ownership of the way the sale of the organization together, but through the interdependence, complementary division of labor, mutual benefit And the more flexible relationship between the network. In this sense, the growing emergence of a global production network headed by a multinational corporation, an organization of production, is an important organizational innovation in the entire world economy in production and trade because it is compatible with all the advantages of the traditional dual governance model, Thus making the organization of production more efficient and also making a certain enterprise in the network, especially the leading manufacturer, more competitive. Therefore, the advent of a global production network dominated by multinational corporations has formed a new micro-foundation for economic globalization.

\section{Global production integration and industrial upgrading}

At present, with the gradual integration of a large number of industries in developing countries into the network of division of labor in global production, a certain degree of industrial upgrading has been achieved and the capabilities of enterprises participating in the division of labor within the network have been raised. The status in the network is also Rising continuously, the upgrading of the related industries as a whole network has been continuously improved. In the future, enterprises in developing countries should further promote industrial upgrading within their networks by participating in global production networks, and must analyze many influencing factors in detail and continuously seek advantages and avoid disadvantages. In this chapter, the author is based on the theoretical analysis of motivation and determinants of the global production networks in chapter two above. Specifically, the theoretical comparative analysis of static comparative advantages, economies of scale, endogenous comparative advantages, transaction costs, Market competition and many other factors to actualize, with the actual establishment of the global production network upgrade factors affecting the model, combined with specific cases for analysis. These factors include the network governance model, the policy system, the external environment, business characteristics and other four categories.

Since Coase first distinguished the two ways in which markets and firms can coordinate deals with the notion of transaction costs, the new institutional economists have for quite some time been focusing on the substitution between the two types of organization, internal organization and market transactions Relationships and their governance patterns. According to Hart and Grossman, the transactions with low asset specificity and transaction frequency adopt entirely random market transactions. The market governance model based on the contract, property rights and price mechanism has high specificity and high transaction frequency The transaction uses a fully internalized business transactions, corresponding to the hierarchical level of internal corporate governance model instead of the market contract. However, as Williamson said, there are in fact a large number of intermediary and intermediate trading organizations between the two extreme modes of coordination of the market and the enterprise.

Modular production networks often involve the production of more complex products, but their manufacturing standards or related knowledge are also easier to code and the production technology is standardized, and the product structure has a modular character. The suppliers are capable of providing all products or parts of modules, Coupled with the internal control of coding information more difficult, so the degree of asset specificity is lower, leading manufacturers need to be regulated and controlled. However, since knowledge is encoded, there is not much explicit coordination of knowledge-related issues, and as with every market in a modular production network, the cost of moving to a new trading partner is very low, as in the case of market transactions. The biggest advantage of modular production network is the rapid and flexible response to the market, and for the network manufacturers, especially leading manufacturers, lower input costs. In the relational 
production network, product standards are not easy to be encoded, and the trading relationships among manufacturers are complicated. However, suppliers have stronger capabilities and frequent knowledge exchange between suppliers and leading vendors, especially the face-to-face exchange of information with each other Extremely frequent, explicit coordination of the leading vendors in this process will play a big role, with a high level of interdependence based on credit, social or geographical linkages, family and ethical relationships, etc., and as a result, both turn The costs of other trading partners are higher. Compared with the market-based and modular production networks, the power relationship between the two parties is relatively equal.

In the leading production network, the coding of knowledge is easier, but the suppliers have lower supply capacity, forcing the leading manufacturers to intervene and control them. In this process, the leading manufacturers will invest more resources and pay more Efforts will therefore lock their suppliers in their own production networks and exclude other leaders from entering. On the other hand, due to the high transfer costs, the production activities, such as simple assembly, which are limited to a relatively small area of production depend on the design, logistics, parts procurement and production technology upgrades of the leading manufacturers. Despite the asymmetric power of both parties, a leading production network can reduce opportunistic behavior between the two parties and at the same time provide suppliers with a product market exit. In the hierarchical production network, because of the complexity of the product, the production standard is difficult to be encoded. In addition, the supplier's ability is lower, and the production process can only be completed within the enterprise. At the same time, explicit knowledge can also be controlled within the network for exchange, And effectively manage complex production networks and effectively control resources, especially the control of intellectual property. In this case, explicit coordination activities by leading vendors are more involved. Of course, the power of leading vendors and suppliers in the network Status is also extremely asymmetrical. What needs to be pointed out is that the governance modes of the above-mentioned five production networks are not static with each other but can be transformed into each other dynamically. What kind of network governance mode a multinational company decides to adopt depends on its interest in outsourcing The trade-off of risk. The above five kinds of network management model can use the following form to make a summary of the specific circumstances.

\section{Chinese industrial upgrading strategy}

In building an independent innovation system that is directed by industrial clusters that are involved in intra-network division of labor, we must adhere to the principle of openness, that is, to establish an open system of independent innovation, which is of crucial importance to industries that are already deeply embedded in global production networks. Specific measures for an independent innovation system may include formulating the development strategy of industrial clusters participating in the global division of production networks. Strategic positioning and industrial guidance system Accelerating the establishment of a technological innovation system that is mainly based on the enterprise and closely integrated with production, teaching and research to increase investment, optimize structure and build more Channel and multi-level investment and financing system for science and technology Promote informatization of national economy and society and promote the leapfrogging of traditional industries Innovate the technology transfer mechanism Implement the strategy of intellectual property strategy and technology standard system and effectively improve the core competitiveness of the industry Deepen the reform of personnel distribution system, Accelerate the Training of Innovative Talents Vigorously develop cooperation in new science and technology with other countries, exchange innovation and culture, promote the popularization of science, and raise public awareness of science and technology.

In the global production network contains a wealth of knowledge resources, knowledge transfer and organizational learning mechanism, it can be said that the global production network itself is a typical learning organization. For any enterprise in the global production network, creating a learning organization and establishing a management mechanism corresponding to the global production network are all necessary for making full use of the knowledge resources in the global 
production network and coordinating with the global production network and upgrading continuously condition. Therefore, among the processing enterprises, especially those enterprises that have reached a certain scale, the management philosophy and management mode of the learning organization are implemented to enhance the learning ability and learning efficiency of the enterprises themselves and the related domestic production networks and form a learning domestic production network and Learning industrial clusters have very important significance. As to the connotation of learning organization, scholars in different countries have given different understandings according to their own theory and thinking. Generally believed that learning organization has a strong organizational learning ability, the interaction between the organization and the environment can quickly learn to grow. As for how to create a learning processing and manufacturing enterprise in the context of a global production network, here is a basic framework for reference from the previous research. The framework consists of preparation, learning, application and evaluation of interrelated phases. It should be noted that processing enterprises located in global production networks pay special attention to the characteristics and changes of global production networks in various stages of establishing a learning organization, their position in the global production network and the interaction between this enterprise and the global production network.

In recent decades, the concept of network has become very popular in academia and widely used in practice. Compared with other organizational forms, the concept of network has shown a clear comparative advantage. There is currently no single concept of what constitutes a network. It is usually associated with a loose coalition and generally refers to the relatively loose relationship established between different actors in pursuit of certain common goals and interdependencies. However, once established, subjects generally have the characteristics of interdependence, intensive communication, mutual benefit and high trust. Of course, occasional conflicts and power asymmetries can not be ruled out Sex. The establishment of a domestic production network as mentioned here is centered on the leading manufacturing and manufacturing enterprises embedded in the global production network and extensively absorbing relevant enterprises to establish a loose consortium or cooperation. The formation and extension of the domestic production network can make the organizations engaged in global division of work and production more adapted to the operation mechanism of global production networks and at the same time can take full advantage of the advantages of both network management organizations and the two governance modes of the market which are beneficial to processing enterprises The formation and improvement of core competitiveness also help the value chain to expand in China, the growth of related enterprises and the development of supporting industries.

\section{Conclusion}

This paper focuses on analyzing and discussing the theoretical basis of the motivation and development of global production networks and the upgrading mechanisms brought by global production networks to related industries in China, including the mechanism of knowledge transfer and diffusion within networks, the connotation and upgrading of industrial upgrading within networks Mechanism, the model framework of the influencing factors of industrial upgrading in the network, the empirical analysis of the status quo in Chinese related industrial networks and the industrial upgrading countermeasures based on the global production network model.

\section{References}

[1] Li Guoxue, Zhang Yuyan. Asset-Specific Investment, Global Production Network and Industrial Structure Upgrading in China [J] .World Economy Research. 2010 (05)

[2] Zhao Wei, Jiangdong.Journal of Zhejiang University (Humanities and Social Sciences). 2010

[3] Jiang Qianqian. An Empirical Study of Zhejiang Foreign Trade Market Structure Based on HHI 
[J] .Reformation \& Strategy. 2010 (01)

[4] Xu Yi, Zhang Erzheng. FDI, Outsourcing and Technological Innovation: An Empirical Study Based on Input-Output Table Data [J]. World Economy .2008 (09)

[5] Xu Yingzhi, Jin Naili, Sun Jian. Technological Progress, Boundary of Enterprises and Outsourcing Decision-making - An Empirical Study Based on Chinese Manufacturing Data [J] .Chinese Economic Journal. 2008 (05)

[6] Zhang Qiuju, Zhu Zhongdi. Empirical Analysis on the Relationship between the Undertaking of Transnational Outsourcing and the Technological Progress in China - Based on VECM's Short-term and Short-term Causality Test [J] .World Economy Research. 2008 (06)

[7] Fan Ying. Theoretical Development of Heterogeneous Business Model [J]. International Trade Issues. 2008 (03) 